The Astrophysical JouRnal, 480:188-195, 1997 May 1

(C) 1997. The American Astronomical Society. All rights reserved. Printed in U.S.A.

\title{
EXTREME MICROLENSING TOWARD THE GALACTIC BULGE
}

\author{
ANDREW GOULD ${ }^{1}$ \\ Department of Astronomy, Ohio State University, Columbus, OH 43210; gould@payne.mps.ohio-state.edu \\ Received 1996 March 28; accepted 1996 November 8
}

\begin{abstract}
Extreme microlensing events, defined as events with maximum magnification $A_{\max } \gtrsim 200$, are a potentially powerful probe of the mass spectrum and spatial distribution of objects along lines of sight toward the Galactic bulge. About $30 \mathrm{yr}^{-1}$ such events are expected for main-sequence sources with $I_{0}<19$. For many of these it is possible to measure both a "proper motion" and a "parallax," that together would yield individual mass, distance, and transverse-speed determinations of the lensing object. The proper motion is determined from finite-source effects when the lens transits, or nearly transits, the source. The parallax is determined by observing the difference in the light curve as seen from two Earth observatories separated by about 1 Earth radius, $R_{\oplus}$. The size of the parallax effect is $\sim A_{\max } R_{\oplus} / \tilde{r}_{e}$, where $\tilde{r}_{e}$ is the projected Einstein radius and can be of order $1 \%$. Detection of candidate events requires a pixellensing search of the entire bulge once per day, preferably by at least two observatories on different continents. Follow-up observation must be carried out using optical/infrared photometry, with short (e.g., 1 minute) exposures on small $(\gtrsim 1 \mathrm{~m})$ telescopes. Extreme microlensing observations toward the Large Magellanic Cloud do not appear feasible at the present time.
\end{abstract}

Subject headings: gravitational lensing - stars: statistics

\section{INTRODUCTION}

Four groups are presently searching for microlensing events toward the Galactic bulge: OGLE (Udalski et al. 1994), MACHO (Alcock et al. 1995a, 1997a), DUO (Alard 1996), and EROS (Augbourg et al. 1995; M. Spiro 1996, private communication). Events detected in this direction probe the mass content of the Galactic disk (Paczyński 1991; Griest et al. 1991) as well as the bulge itself (Kiraga \& Paczyński 1994). For microlensing by a point source, the observed flux $F(t)$ from a lensed source star is given by $F(t)=F_{0} A(t)$ where $F_{0}$ is the flux of the unlensed source and (Paczyński 1986)

$$
A[x(t)]=\frac{x^{2}+2}{x\left(x^{2}+4\right)^{1 / 2}}, \quad x(t)=\left[\omega^{2}\left(t-t_{0}\right)^{2}+\beta^{2}\right]^{1 / 2} .
$$

Here $\omega^{-1}$ is the timescale of the event, $t_{0}$ is the time of maximum, and $\beta$ is the impact parameter normalized to the angular Einstein radius, $\theta_{e}$,

$$
\theta_{e}=\left(\frac{4 G M}{c^{2} D}\right)^{1 / 2}, \quad D \equiv \frac{D_{\mathrm{ol}} D_{\mathrm{os}}}{D_{\mathrm{ls}}},
$$

where $M$ is the mass of the lens and $D_{\mathrm{ol}}, D_{1 \mathrm{~s}}$, and $D_{\mathrm{os}}$ are the distances between the observer, lens, and source. Of the three lensing parameters which can be extracted from a lensing event (see eq. [1.1]), only the timescale is related to the physical parameters of the lens,

$$
\omega=\frac{v}{D_{\mathrm{ol}} \theta_{e}},
$$

where $v$ is the transverse speed of the lens relative to the observer-source line of sight. The other two parameters, $t_{0}$ and $\beta$, simply reflect the geometry of the event.

\footnotetext{
${ }^{1}$ Alfred P. Sloan Foundation Fellow.
}

One would like to use the observed lensing events to learn about the details of the lens population. For example, is this population fully accounted for by the known populations of luminous stars? What is the mass spectrum of the lenses? What is their distribution along the line of sight? What are their kinematic properties? Because the one observable $\omega$ is a complicated combination of the physical properties of the lens, it is difficult to obtain unambiguous answers to these questions. Zhao, Spergel, \& Rich (1995) and Han \& Gould (1996) estimated the mass spectrum from the observed distribution of timescales by assuming that the sources and lenses have velocity and spatial distributions like those of observed stars. Han \& Gould (1996) found that the inferred mass spectrum is inconsistent at the $5 \sigma$ level with that of nearby stars as determined by Gould, Bahcall, \& Flynn (1996) using Hubble Space Telescope (HST) observations. If confirmed by continuing observations, this would be an extremely intriguing result. Nevertheless, the approach adopted is fundamentally limited both by its statistical nature and by its dependence on unverifiable assumptions about the phase space distribution of the lenses. One would like to be able to measure $M, D_{\mathrm{ol}}$, and $v$ for each individual lens, or at least for a representative subsample of events.

It would be possible to determine individual masses provided one could somehow measure $\theta_{e}$ (eq. [1.2]) and $\tilde{r}_{e}$, the Einstein radius projected onto the observer plane,

$$
\tilde{r}_{e}=D \theta_{e}=\left(\frac{4 G M D}{c^{2}}\right)^{1 / 2} .
$$

From equations (1.2) and (1.4), one finds

$$
M=\frac{c^{2}}{4 G} \tilde{r}_{e} \theta_{e} .
$$

In fact, since $D_{\text {os }}$ is typically known to within $\sim 10 \%$ simply from the source's membership in the bulge, one also gets a good estimate of the position and transverse speed of the 
lens,

$$
D_{\mathrm{ol}}=\left(\frac{1}{D_{\mathrm{os}}}+\frac{\theta_{e}}{\tilde{r}_{e}}\right)^{-1}, \quad v=\frac{\omega}{\tilde{r}_{e}^{-1}+\left(\theta_{e} D_{\mathrm{os}}\right)^{-1}} .
$$

The determination of $\tilde{r}_{e}$ is often called a "parallax measurement" because it is found by observing the lensing event from two different positions in the observer plane. The determination $\theta_{e}$ is often called a "proper motion measurement" because the product $\mu=\omega \theta_{e}$ is the angular speed of the lens relative to the source.

There is no lack of ideas for measuring parallaxes and proper motions for special rare classes of events. For example, for long events the position of Earth changes enough during the event to allow a parallax measurement (Gould 1992; Alcock et al. 1995b). However, while the long events are an interesting subclass, they are by definition unrepresentative of the lenses as a whole. Moreover, parallax measurements do not by themselves permit determination of the mass without a simultaneous proper motion measurement, and the fraction of long events for which such measurements are possible is small. To be useful as probes of the lens mass spectrum, what is required is that both quantities be measured for a representative sample of events.

One approach is to obtain parallaxes using a satellite in solar orbit (Refsdal 1966; Gould 1994b, 1995b; Gaudi \& Gould 1997) and proper motions from finite source effects for small $\theta_{e}$ and from optical interferometry for large $\theta_{e}$. With next generation instruments, this approach could yield $\sim 35$ mass measurements per year with no serious selection bias (Gould 1996b).

Here I discuss another approach that, while substantially less effective than the one just described, could be initiated much earlier.

\section{EXTREME MICROLENSING EVENTS}

The basic idea of this paper is to measure both $\tilde{r}_{e}$ and $\theta_{e}$ for a very special yet nearly representative subclass of events: the extreme magnification events (EMEs). EMEs are events with maximum magnifications

$$
A_{\max } \gtrsim Q,
$$

where $Q$ is a large number, typically $Q \sim 200$. For equation (2.1) to hold, two physical conditions must be satisfied:

$$
\beta \lesssim Q^{-1}, \quad \theta_{*} \lesssim Q^{-1} \theta_{e},
$$

where $\theta_{*}$ is the angular radius of the source star. The first condition restricts the geometry of the event, while the second restricts the class of source stars. The value of $Q$ (i.e., the selection function) has a well-understood dependence on the physical characteristics of the lens, which accounts for the above description of EMEs as "nearly representative" (see $\S 3$ ).

\subsection{EME Parallaxes}

Because of parallax, microlensing events appear slightly different when viewed from different observatories on Earth (Holz \& Wald 1996). Just as with satellite parallaxes (Gould 1994b), the events will have different impact parameters $\beta$ and $\beta^{\prime}$ and different times of maximum $t_{0}$ and $t_{0}^{\prime}$. The difference can be combined into a single vector $\Delta \boldsymbol{x}$,

$$
\Delta \boldsymbol{x}=(\omega \Delta t, \Delta \beta),
$$

where $\Delta t \equiv t_{0}^{\prime}-t_{0}$ and $\Delta \beta \equiv \beta^{\prime}-\beta$. Let the separation between the observatories (projected onto the plane, per- pendicular to the line of sight) be $d_{\text {sep }}$. Then, if $\Delta x$ can be measured, one can determine $\tilde{r}_{e}$ from

$$
\tilde{r}_{e}=\frac{d_{\text {sep }}}{\Delta x}, \quad \Delta x \equiv|\Delta x| .
$$

Of course, since typically $\tilde{r}_{e} \sim O(\mathrm{AU})$ and $d_{\text {sep }} \sim R_{\oplus}$ where $R_{\oplus}$ is the radius of the Earth, $\Delta x$ is incredibly small: $\Delta x \sim$ $R_{\oplus} / \mathrm{AU} \sim 1 / 25,000$. Not surprisingly, the microlensing community greeted this suggestion with some skepticism, and Holz \& Wald (1996) themselves made no claims that the effect could actually be observed, only that photon statistics alone do not preclude such observations.

For EMEs, however, such Earth-based parallaxes are within the range of present capabilities. This is because the observable effects do not scale as $\Delta x$, but as $\Delta x / x$. Since $x \sim Q^{-1}$ near the peak, EME parallax effects are $O(1 \%)$. To make a simple quantitative analysis, I assume that the photometry has a fixed fractional accuracy $\sigma$ and that the errors are uncorrelated. (In fact, correlations among the errors may pose significant problems. I discuss these problems in the Appendix.) The event is observed from $t_{0}-T$ to $t_{0}+T$ at a rate $N \omega \beta^{-1}$. That is, the observations are carried out $N$ times per "effective timescale," $t_{\text {eff }}$, where

$$
t_{\text {eff }} \equiv \frac{\beta}{\omega} \text {. }
$$

I then find that the errors $\delta t_{0}$ and $\delta \beta$ in the measurements of $t_{0}$ and $\beta$ are given by (e.g., Gould 1995a),

$$
\begin{aligned}
\frac{\delta t_{0}}{t_{\text {eff }}} & =\frac{\sigma}{\{N[\Phi-\sin (2 \Phi) / 2]\}^{1 / 2}}, \\
\frac{\delta \beta}{\beta} & =\frac{\sigma}{\{N[\Phi+\sin (2 \Phi) / 2]\}^{1 / 2}}, \\
\tan \Phi & \equiv \frac{T}{t_{\text {eff }}} .
\end{aligned}
$$

Hence, the rms errors in the measurement of $\Delta x$ are

$$
\delta \Delta x \sim N^{-1 / 2} \beta \sigma F(\Phi), \quad[F(\phi)]^{2}=\frac{2}{\Phi}\left[1-\left(\frac{\sin 2 \Phi}{2 \Phi}\right)^{2}\right]^{-1} \text {. }
$$

For simplicity, I henceforth assume that the observations can be carried out for a duration $\sim 4 t_{\text {eff }}$, so that $F(\Phi) \sim 1.45$. For typical events seen toward the bulge, $\omega^{-1} \sim 10$ days (Alcock et al. 1995a). Hence, the effective timescale for an EME with $\beta^{-1} \sim 200$ is $t_{\text {eff }} \sim 1 \mathrm{hr}$. Assuming one could make one observation per minute each with fractional accuracy $\sigma=1 \%$, then $\delta \Delta x \sim \beta / 540 \sim$ $10^{-5}$. Recall that the typical scale of interest is $\Delta x \sim$ $R_{\oplus} /$ AU $\sim 4 \times 10^{-5}$. For lower $\beta$, but the same accuracy and rate of observations, the precision improves $\propto \beta^{1 / 2}$.

These results show that Earth-based parallax measurements of EMEs are generally within the range of present technology.

\subsection{EME Proper Motions}

When the lens transits the source, the light curve deviates from the point-source form (1.1). One can then measure $x_{*}$,

$$
x_{*} \equiv \frac{\theta_{*}}{\theta_{e}},
$$


the value of $x$ when transit occurs (Gould 1994a; Nemiroff \& Wickramasinghe 1994; Witt \& Mao 1994). If $\theta_{*}$ is known (as it usually is from the dereddened color and magnitude and Stefan's law), then one can determine $\theta_{e}=\theta_{*} / x_{*}$. If the lens comes close to the source but does not transit, there is still a fractional deviation from the point-source formula $\Delta A / A \sim(\Lambda / 8)\left(x_{*} / x\right)^{2}$, where $\Lambda$ is the second radial moment of the source normalized so that $\Lambda=1$ for a uniform disk. Unfortunately, with single-band photometry one cannot put this effect to use because it cannot be distinguished from a slight shift in $\beta$ (Gould \& Welch 1996). However, since stars are limb-darkened by different amounts in different bands, near transits give rise to color effects that can be measured (Witt 1995). Specifically, Gould \& Welch (1996) find $\Lambda^{H}-\Lambda^{V}=0.07$, allowing measurement of $\theta_{e}$ for $\beta \lesssim$ $2 x_{*}$. Since EMEs typically fall in or near this range, it should often be possible to measure their proper motions.

\subsection{Combined Parallaxes and Proper Motions}

At first sight, it may appear that the very condition required to measure $\theta_{e}$ (transit or near transit of the source) would make measurement of $\tilde{r}_{e}$ impossible. In fact, the majority of mass measurements are not severely affected by this potential problem. Consider first an event with $\beta=1 / 200$ and $x_{*}=1 / 300$. At the peak of the event, the perturbation due to finite size is $\Delta A / A=(\Lambda / 8)\left(x_{*} / \beta\right)^{2} \sim 5 \%$ (where I have assumed $\Lambda=0.9$ ). Since this is several times the change in $A$ due to parallax $(\sim \Delta \beta / \beta \sim 1 \%$ ), one might worry that it would render the parallax shift unobservable. In fact, since the finite-size effect (at fixed source-lens separation) is identical for the two observers, the difference in their observed maximum magnifications still accurately measures $\Delta \beta$. The finite-source effect would lead to $\sim 5 \%$ fractional error in the estimate of $\Delta \beta / \beta$ if left uncorrected, but even the correction due to this minor systematic error is not difficult to determine once the size of the source is measured.

If the lens actually transits the source, $\beta<x_{*}$, then the situation is more complicated. In this case, one could restrict attention to those portions of the light curve where $x \gtrsim x_{*}$, for which the light curve is either unaffected by finite-source effects or the corrections due to these effects are well determined. (As in the previous example, one is interested only in the difference between the two curves, so the corrections play a minor role.) I assume in this case that there are $N$ measurements per stellar crossing time $t_{*} \equiv$ $x_{*} / \omega$, each with accuracy $\sigma$, and that the measurements are carried out over a symmetric interval of half width $T$. I then find (see, e.g., Gould 1995a)

$$
\begin{aligned}
& \frac{\delta t_{0}}{t_{*}}=\frac{\sigma}{N^{1 / 2}}\left\{\frac{x_{*}}{\beta}\left[\left(\Phi_{f}-\Phi_{i}\right)-\left(\frac{\sin 2 \Phi_{f}}{2}-\frac{\sin 2 \Phi_{i}}{2}\right)\right]\right\}^{-1 / 2}, \\
& \frac{\delta \beta}{x_{*}}=\frac{\sigma}{N^{1 / 2}}\left\{\frac{x_{*}}{\beta}\left[\left(\Phi_{f}-\Phi_{i}\right)+\left(\frac{\sin 2 \Phi_{f}}{2}-\frac{\sin 2 \Phi_{i}}{2}\right)\right]\right\}^{-1 / 2}
\end{aligned}
$$

where $\tan \Phi_{f} \equiv \omega T / \beta$ and $\cos \Phi_{i} \equiv \beta / x_{*}$. For $\beta \ll x_{*}$, these equations have the limiting forms

$$
\frac{\delta t_{0}}{t_{*}} \rightarrow \frac{\sigma}{(2 N)^{1 / 2}} \text { and } \frac{\delta \beta}{x_{*}} \rightarrow \frac{\sigma}{(2 N / 3)^{1 / 2}} \frac{x_{*}}{\beta} \quad\left(\beta \ll x_{*}\right) .
$$

Equation (2.11) shows that if $\Delta t$ can be measured in a marginal transit event $\left(\beta=x_{*}\right)$ with a given accuracy, then approximately the same accuracy can be achieved for all transit events $\left(\beta<x_{*}\right)$. However, the accuracy of the measurement of $\Delta \beta$ deteriorates linearly with impact parameter as the impact parameter falls well below the source size. In $\S 6$, I discuss the possibility of compensating for this loss of information about $\Delta \beta$ by making observations from a third site.

\subsection{Marginal Transit Events Are Optimal}

From the foregoing discussion, it is clear that the best events are those for which the lens just transits the limb of the star, $\beta=x_{*}$. For larger $\beta$, the parallax effect declines inversely as $\beta$, and for $\beta>2 x_{*}$, the proper motion cannot be measured. On the other hand, for smaller $\beta$, the measurement of $\Delta \beta$ becomes more difficult. Even if one compensates for this problem by making observations from a third site (see § 6) parallax measurements are still no more precise than for marginal transits. Thus, marginal transit events allow us to understand the fundamental limits of the technique.

The maximum parallax effect occurs at transit and is given by $\Delta x / x_{*}$, which may be evaluated as

$$
\frac{\Delta x}{x_{*}}=\frac{d_{\mathrm{sep}} / \tilde{r}_{e}}{\theta_{*} / \theta_{e}}=\frac{d_{\mathrm{sep}}}{R_{*}} \frac{D_{\mathrm{ls}}}{D_{\mathrm{ol}}},
$$

where $R_{*}=D_{\mathrm{os}} \theta_{*}$ is the physical radius of the source, and where in the last step I have made use of $\tilde{r}_{e}=D \theta_{e}$ from equations (1.2) and (1.4). As I show in $\S 4$, the typical source stars for EMEs are solar-type stars or slightly fainter. Assuming the observatories are about 1 Earth radius apart, equation (2.12) becomes

$$
\frac{\Delta x}{x_{*}}=\frac{R_{\oplus}}{R_{\odot}} \frac{D_{1 \mathrm{~s}}}{D_{\mathrm{ol}}} \sim 0.01\left(z^{-1}-1\right),
$$

where $z \equiv D_{\mathrm{ol}} / D_{\mathrm{os}}$ is the fractional distance of the lens to the source. Hence to measure the mass of a disk lens $(z \sim 0.5)$ requires detection of a $1 \%$ effect and to measure the mass of a bulge lens $(z \gtrsim 0.75)$ requires detection of a $\lesssim 0.3 \%$ effect. While the exact threshold of the experiment cannot be determined without a better understanding of the limits to the photometric accuracy, it is clear that bulge events with sufficiently small lens-source separation will be beyond the limit. I call this limit $z_{\max }$. I discuss the effect of this limit on the selection function in $\S 3$, and possible methods for extending it in $\S 5$.

\section{SELECTION FUNCTION}

Let $\mathscr{S}\left(M, D_{\text {ol }}, z\right)$ be the fraction of lensing events with parameters $M, D_{\mathrm{ol}}$, and $z=D_{\mathrm{ol}} / D_{\mathrm{os}}$ that have measurable parallaxes and proper motions. As discussed at the end of the previous section, the measurement precision sets a limit $z_{\max }$ such that parallax cannot be measured for $z>z_{\max }$. The next most important selection effect is that, to measure proper motions, the impact parameter must satisfy $\beta<2 x_{*}$. That is, $\mathscr{S} \propto \theta_{*} / \theta_{e}$. Since parallax measurements generally require small source stars, I initially assume that $\theta_{*}$ is fixed. (I relax this assumption below.) Hence,

$$
\mathscr{S}\left(M, D_{\mathrm{ol}}, z\right) \propto \theta_{e}^{-1} \Theta\left(z_{\max }-z\right) \propto\left(\frac{M}{D}\right)^{1 / 2} \Theta\left(z_{\max }-z\right),
$$


where $\Theta$ is a Heaviside (unit) step function.

While equation (3.1) is important for understanding the relation between the events with measured masses and the full ensemble of events, it is not the most useful form of the selection function. What is fundamentally of interest is not the distribution of parameters for the ensemble of lenses, but the distribution for the underlying populations of objects that give rise to the events. The lensing events are themselves a biased sample of the underlying population. They occur with relative frequency $\mathscr{F}$ proportional to their (one-dimensional) cross section and transverse speed, i.e., $\mathscr{F} \propto \theta_{e} D_{\mathrm{ol}} v$. Hence, the fraction of all objects whose mass can be measured is

$$
\mathscr{F} \times \mathscr{S} \propto D_{\text {ol }} \bar{v}\left(D_{\text {ol }}\right) \Theta\left(z_{\text {max }}-z\right),
$$

where $\bar{v}\left(D_{\mathrm{o} 1}\right)$ is the mean transverse speed of objects at distance $D_{\mathrm{ol}}$. For the simplest models (see, e.g., Fig. 8 from Han \& Gould 1995), one expects $\bar{v} \propto D_{\text {ol }}$, in which case $\mathscr{F} \times \mathscr{S} \propto D_{\text {ol }}^{2} \Theta\left(z_{\max }-z\right)$. This result implies that EME mass measurements heavily favor more distant populations, until the limit of parallax detection is reached close to the bulge. It therefore emphasizes the importance of pushing that limit as far as possible (see $\S 5$ ).

While equation (3.2) reflects the most important selection effects, there are other effects which induce some additional minor modifications. First, higher mass lenses are slightly favored relative to equation (3.2) (which has no mass dependence). To see this, consider two masses with $M_{1}=$ $4 M_{2}$, both at the same distance $D_{\mathrm{ol}}$. For illustration, assume that the parallax and proper motion of $M_{2}$ are only just measurable when $\beta_{2}=2 x_{*}=2 \theta_{*} / \theta_{e, 2}$ for a fiducial source star, $I_{0}=19$. In the above analysis, it was assumed that for the same star, the larger mass would have measurable proper motion only if $\beta_{1}<\theta_{*} / \theta_{e, 1}$, which is half as great. This is true, provided the source is the same. However, if $M_{1}$ were lensing a source star with 2 times the radius of the fiducial source, proper motions would be measurable to 2 times the impact parameter. Such larger stars are accessible to $M_{1}$ (but not $M_{2}$ ) because $\theta_{e, 1}$ is larger and so the parallax effect is larger at fixed angular separation.

The reason that this is not a major effect is the steepness of the luminosity function, which scales inversely with luminosity when binned in magnitude intervals. Assuming that all stars have the same temperature (which is approximately true near the turnoff), then stars with two times greater radius are four times less numerous. Hence, even for more massive lenses, most of the events with measurable proper motions will be near the magnitude limit.

A similar effect also favors nearby lenses because these also have larger Einstein rings: $\theta_{e} \propto D^{-1 / 2}$. The effect is likewise small.

\section{EVENT RATE AND DETECTION STRATEGY}

A fraction $Q^{-1}$ of all events have $A_{\max }>Q$, where I have for the moment ignored finite-size effects. Since the present detection rate is $O(100) \mathrm{yr}^{-1}$, this would seem to imply that there would be $\lesssim 1$ event per year for $Q \sim 200$. However, the present detection strategy is not optimized for finding EMEs. Here I show how an aggressive search could yield $\sim 30$ EMEs per bulge season.

Consider a main sequence star in the bulge with $I_{0} \sim 19$. If this star were magnified by a factor $A \sim 200$, it would have a dereddened apparent magnitude $I_{0, A}=200=13.2$, i.e., it would be brighter than most clump giants. Hence, at least near the peak, it would be as easy as a giant to photometer. One could hope to achieve $1 \%$ photometry or even better on such stars. Suppose that the star lay behind several magnitudes of extinction. The photometry problems induced by crowding would not change relative to the extinction-free case since all neighbors would suffer the same extinction. The photometry would be degraded only if there were insufficient photon statistics. Assuming 1" seeing and a sky brightness of $I=19.6 \mathrm{mag} \operatorname{arcsec}^{-2}$, photon statistics predict errors of $\lesssim 1 \%$ for a 1 minute exposure on a $1 \mathrm{~m}$ telescope at $I=17$. As $I$ have earlier discussed (Gould $1995 \mathrm{c}$ ), there are $\sim 10^{7}$ giants $\left(I_{0}<15\right)$ over an $82 \mathrm{deg}^{2}$ area of the bulge with extinction $A_{I}<3.5$. Using the bulge luminosity function measured by Light, Baum, \& Holtzman (1996), I estimate that there are $N \sim 4 \times 10^{8}$ stars with $I_{0}<19$ in the same region. Assuming an average optical depth $\tau \sim 3 \times 10^{-6}$ (Alcock et al. 1997a), and a mean timescale $\left\langle\omega^{-1}\right\rangle \sim 20$ days, this leads to an estimate of $2 \pi^{-1} Q^{-1} N \omega T_{\text {bulge }} \tau \sim 30 \mathrm{yr}^{-1}$ events for $Q=200$, assuming a bulge season of $T_{\text {bulge }}=180$ days.

It is clearly impossible to identify these events using current search techniques that rely on following the light curves of stars recognized as such in a template image. Since the templates contain few if any of the $I_{0}=19$ stars, lensing events of such stars cannot be detected. Instead, one must make a pixel-lensing search of the type currently being carried out toward M31 (Crotts 1992; Baillon et al. 1993; Tomaney \& Crotts 1996). In M31, there are many unresolved stars per pixel. One therefore subtracts a reference image from the current image to find changes in the brightness of individual stars. These changes appear as isolated point-spread functions (PSFs) on an otherwise flat difference frame. In M31, pixel lensing is the only way to search for lensing events because there are essentially no resolved stars. On the other hand, pixel lensing has not seemed necessary in the bulge or the Large Magellanic Cloud (LMC) because these fields contain many resolved stars. Note, however, that Melchior (1995) has made an initial attempt to find lensing events of unresolved stars in the LMC using pixel lensing, and efforts are continuing to develop this technique in fields with both resolved and unresolved stars. Pixel lensing is not as simple for the bulge as it is for M31 because the resolved stars in the field leave significant residuals in the difference images. To understand this problem concretely, consider a lensing event of an $I_{0}=$ 19 source with $\omega^{-1}=10$ days that is destined to become an EME. One day before maximum, it will have $I_{0, A}=10=$ 16.5. While still about 5 times fainter than a giant, it would be substantially brighter than the net residuals from giants and of course would have a characteristic PSF shape which the residuals would not. Thus, it is likely that it could be recognized assuming that there was a high enough signalto-noise ratio. For the most heavily extincted regions under consideration, $A_{I}=3.5$, the star would have $I=20$, and so would be detectable with signal-to-noise ratio $\sim 25$ if it were on a blank field (assuming 5 minute observations on a $1 \mathrm{~m}$ telescope in $1^{\prime \prime}$ seeing). Whether it could actually be detected amidst the bulge-star residuals would depend on how well the subtraction worked. In any case, events in regions with $A_{I}<2.5$ would very likely be detectable, and these include most of the available bulge field.

In brief, an aggressive pixel lensing search with a $1 \mathrm{~m}$ telescope and a 1 deg $^{2}$ camera, such as now is being com- 
missioned by the EROS collaboration (M. Spiro 1996, private communication), could cover the bulge each night with adequate depth to detect most events, weather permitting. There would be a substantial improvement in the detection rate if the bulge were covered from two continents. In this case one would benefit not only from reduced weather-induced gaps, but would also be more likely to expose when the object was bright enough to be detected but had not yet reached maximum. However, substantial improvements in the speed and efficiency of the real-time alert system would be required to enable the follow-up observations to begin before maximum.

If candidates are selected once per day, then, as discussed above, events destined to become EMEs may have magnifications as small as $A_{\min } \sim 10$. Any "new star" on the image with magnification $A>A_{\text {min }}$ (as estimated from its color and apparent magnitude) must therefore be monitored sufficiently to determine if it is an EME. There will be $\sim N \tau A_{\min }^{-2} \sim 12$ such events on any given night. Of these, less than one per night will have $A \gtrsim 50$, so that observations will require aggressive attention. The remainder can be monitored once every few hours to determine if they are becoming high magnification events. This should require less than one hour per night of observations by the entire network of follow-up observatories (see $\S 5$ ) and therefore should not interfere unduly with other programs of observation. The rate of genuine alerts $(\sim 12$ per night) then sets the scale for allowable false alarms due to reduction errors. If these exceed the number of genuine alerts, they would compromise or perhaps overwhelm the follow-up network. If false alerts become a problem, the search would have to be restricted to the southern half of the Galactic bulge where the extinction is generally $A_{I} \lesssim 2$ and consequently events seen at $A \sim A_{\min }$ have $I \lesssim 18.5$ and are hence easily distinguished by eye on a 5 minute exposure.

\section{FOLLOW-UP PHOTOMETRY}

To obtain both parallaxes and proper motions, accurate photometry is required in two bands, preferably one optical and one infrared. The reason is that parallax measurements deteriorate rapidly for $\beta<x_{*}$, while proper motion measurements are impossible for $\beta>x_{*}$ unless there is photometry in two bands. It is possible to evade the parallax measurement problem that arises at low impact parameters, but as I discuss in $\S 6$, this evasion itself introduces significant logistical difficulties. Hence, the first requirement is to put specialized cameras equipped with dichroic beamsplitters (preferably optical/infrared) on telescopes dedicated to microlensing follow-up observations on several continents.

There are already two networks of observers currently engaged in follow-up photometry of ongoing microlensing events seen toward the bulge, PLANET (Albrow et al. 1996) and GMAN (Pratt et al. 1996). The primary objective of these networks is to find light-curve deviations that would be the signature of planets (Mao \& Paczyński 1991; Gould \& Loeb 1992). Like the EME observations proposed here, the planet searches require quick response to alerts and a high frequency of observations, and planet searches would benefit greatly from optical/infrared photometry (Gould \& Welch 1996). One such camera is already being built and there is an active proposal to build a copy (D. DePoy 1996, private communication). Moreover, there is considerable interest in expanding the planet search. Since the planet search and the EME follow-up require similar instruments and modes of observation, it would be natural to combine the two.

A major goal of the follow-up photometry is to minimize the errors. Recall from $\S 2.4$ that one typically expects the size of the parallax effect to be $\Delta x / x_{*} \sim 0.01\left(z^{-1}-1\right)$, and recall from $\S 2.1$ that to measure this effect to $\sim 20 \%$ accuracy requires the same order of precision in each 1 minute exposure, i.e., $1 \% \times\left(z^{-1}-1\right)$. Thus, if the measurement accuracy is limited to $\sigma \sim 1 \%$, the mass measurements will reach only to $z_{\max } \sim 0.5$, that is, half way to the Galactic center. If the accuracy is $\sigma \sim 0.3 \%$, then $z_{\max } \sim 0.75$, which would include most disk as well as some bulge events. If $\sigma \sim 0.15 \%$, then events with $D_{1 \mathrm{~s}} \gtrsim 1 \mathrm{kpc}$ will be accessible, which would give good sensitivity to bulge lenses.

The conventional wisdom is that $1 \%$ photometry is the limit for crowded fields, regardless of the signal-to-noise ratio. This view is born of extensive experience with PSF fitting of globular clusters and other crowded fields. Lensing searches have also used PSF fitting, as have all follow-up searches. Measuring the mass of bulge lenses using EMEs will require another approach to photometry. Pixel-lensing techniques may provide the answer to this problem. I mentioned in $\S 4$ that pixel lensing would be required to find the EMEs in the first place. However, the initial pixel-lensing search and the pixel-lensing follow-up observations have very different requirements and very different possibilities. In the initial search, a $10 \sigma$ detection (and hence 10\% photometry) would be quite adequate, while better than $1 \%$ photometry is needed in the follow-up to improve on current techniques. On the other hand, the initial searches are driven to the largest pixel sizes consistent with Nyquist sampling in order to cover the largest angular area in the shortest time. Large pixels seriously degrade pixel-lensing photometry unless, as with the Hubble Space Telescope (HST), the pointing is extremely good (Gould 1996a). The follow-up observations are under no pressure toward large pixels and, in fact, several partners in PLANET and GMAN obtain highly oversampled data. These ongoing follow-up observations would make an excellent test bed for refining pixel-lensing techniques in fields containing resolved stars. If such refinements are successful, mass measurements for EMEs can be extended to lenses closer to the bulge. Otherwise they will be restricted to disk objects.

\section{DEGENERACIES}

EME parallaxes are in principle subject to the same two degeneracies that affect space-based parallaxes. First, the source positions as seen by the two observers can be on the same or opposite side of the lens, which leads to a twofold degeneracy in the size of the Einstein ring (see Figs. $1 a$ and $1 b$ from Gould 1994b). Second, there are two possible orientations of the source motion, which leads to a twofold degeneracy in the inferred direction of the transverse velocity (see Figs. $1 a$ and $1 d$ from Gould 1994b). However, the first degeneracy is almost always resolved for EMEs, and the second can be resolved in some cases but in any event is not important.

To see why the first degeneracy is not a major problem, consider an event generated by an object with $M=0.3 M_{\odot}$, $v=150 \mathrm{~km} \mathrm{~s}^{-1}$, and $D_{\mathrm{ol}} / D_{\mathrm{os}}=0.75$. Suppose that the parallax measurement yields $\Delta \beta / \beta \sim \omega \Delta t / \beta \sim 0.005$, based on the assumption that the source is on the same side of the Einstein ring as viewed by both observers. If the source were 
now assumed to be on opposite sides as viewed by the two observers, then the inferred $\Delta \beta$ would be a factor $\sim 400$ larger, implying a larger $\Delta x$ and hence a smaller $\tilde{r}_{e}$ by a factor of $\sim 280$. Using equation (1.6), one finds that the inferred transverse speed would then be $v \sim 2 \mathrm{~km} \mathrm{~s}^{-1}$ and the inferred distance $D_{\mathrm{o} 1} \sim 20 \mathrm{pc}$. For small distances and speeds, the cumulative event rate distribution is proportional to $v^{3} D_{\mathrm{ol}}^{2}$, so the a priori probability of such an event is extremely low. For the transverse velocity to be so nearly perpendicular to the observatory separation vector that $\Delta \beta /(\omega \Delta t)=400$ is even more improbable. Finally, unless the geometry were exceptionally unfavorable, easily observable effects would be generated by Earth's rotational acceleration $\left(\sim 0.1 \mathrm{~km} \mathrm{~s}^{-1} \mathrm{hr}^{-1}\right)$ over a few hours or its orbital acceleration $\left(\sim 0.5 \mathrm{~km} \mathrm{~s}^{-1} \mathrm{day}^{-1}\right)$ over 1 day. As a practical matter, this form of degeneracy is therefore excluded.

The second form of parallax degeneracy affects only the inferred direction of motion. It is therefore irrelevant to any of the results discussed thus far. The direction of motion could be an interesting quantity. However, if it were to be used to measure the lens motion, one would have to make a measurement of the proper motion of the source. The latter is likely to be $\sim 10 \mathrm{~km} \mathrm{~s}^{-1} \mathrm{kpc}^{-1} \sim 2$ mas $\mathrm{yr}^{-1}$ in each direction and so could be roughly measured with two HST exposures separated by $\sim 10 \mathrm{yr}$.

Resolving the degeneracy in the direction of motion requires observing the event from a third location not collinear with the other two (Gould 1994b). In fact, with three such observatories, one could determine the parallax from the three $t_{0}$ measurements alone, i.e., without any information about the impact parameters. This could be useful for the events where the lens passes well inside the source. Recall from $\S 2.3$ that for such events $\Delta t$ is measurable but $\Delta \beta$ is not.

However, observation from three noncollinear observatories creates substantial logistical difficulties. First, in practice the third observatory would have to be either at the South Pole or in the Northern Hemisphere. If the latter, the period each night when the bulge is observable would be short, and therefore the number of northern observatories required to make routine monitoring possible would be large. Second, if three observatories are required for a measurement, the chance of weather problems is high. There would be substantial value, however, in occasional measurements from a third (northern) observatory. The $\beta$ and $t_{0}$ at this observatory are predicted by the measurements at the other two (up to a twofold degeneracy). The measurement would therefore serve as an external check on the internal errors reported by the two southern observatories.

There is yet another form of degeneracy that could affect these measurements, uncertainty in $\omega$. Near the peak of a high-magnification event, the flux is given by

$$
F(t)=\frac{F_{\max }}{\left[1+\left(t-t_{0}\right)^{2} / t_{\mathrm{eff}}^{2}\right]^{1 / 2}},
$$

where

$$
F_{\max }=\frac{F_{0}}{\beta}, \quad t_{\text {eff }}=\frac{\beta}{\omega} .
$$

Since $\omega$ does not appear in equation (6.1), it cannot be determined from the peak of the event. Since $\theta_{e}=\theta_{*} / \omega t_{*}$ and the empirically determined quantities are $\theta_{*}$ and $t_{*}$, uncertainty in $\omega$ leads to an equal uncertainty in $\theta_{e}$. Parallax measurements are affected similarly.

If the unlensed flux $F_{0}$ were known, then one could determine $\beta$ and hence $\omega$ using equation (6.2) together with the measured $F_{\max }$ and $t_{\text {eff }}$. For lensing events observed to date, one usually assumes that $F_{0}$ is the flux observed from the star after (or before) the event. In fact, this postevent flux may include additional light from a binary companion to the source, from the lens itself, or from a random field star.

For EMEs, the postevent flux cannot be reliably measured from the normal search observations. First, the observations are not deep enough. Second, if there are $4 \times 10^{8}$ source stars over $82 \mathrm{deg}^{2}$, then there are an average of 0.4 sources $\operatorname{arcsec}^{-2}$, making measurements in $\sim 1^{\prime \prime}$ seeing with 0.6 pixels problematic. However, it would be straightforward to measure the postevent flux using the HST planetary camera. By comparing the color of the star after the event with its color at maximum one could detect or rule out the presence of additional light unless it were from a star of very similar color. Stars of similar color (whether in the bulge or the foreground) to these mainsequence sources would likely have similar or greater brightness. Such bright companions would have a significant effect on the structure of the light curve. Finally, binary companions within the Einstein ring would show up in the structure of the light curve (Griest \& Hu 1992; Han \& Gould 1997). Thus, it appears likely that unlensed companions to the source could be either detected or severely constrained.

\section{PARTIAL INFORMATION}

For transit or near-transit events with $z>z_{\max }$, it will be possible to measure $\theta_{e}$, but one can obtain only a lower limit for $\tilde{r}_{e}$. This limit will provide lower limits on the mass and distance through equations (1.5) and (1.6). If, for example, $z_{\max }=0.75$, then one will know that a bulge lens $\left(D_{1 \mathrm{~s}} \lesssim 2 \mathrm{kpc}\right)$ is being detected, but will have only a lower limit on its mass.

Similarly, although the fraction of nearby disk events with near transits and measurable proper motions is small, there will be a much larger fraction with impact parameters of several source radii that still have measurable parallaxes. In this case, there will be an upper limit on $\theta_{e}$ and therefore on the mass and distance.

These limits, while certainly not as valuable as measurements, can be used in concert with mass measurements of other objects to constrain the overall population.

\section{OBSERVATIONS TOWARD THE LMC}

The prospects for extreme microlensing toward the LMC are substantially less favorable than toward the bulge, in part because there are fewer events, and in part because the sources are more distant. I make a rough estimate of these prospects as follows. First, since there is less extinction toward the LMC, I assume that the observations are carried out to a limit $R \sim 23.5$ roughly corresponding (as in the bulge) to solar-type stars. The actual luminosity function of the LMC at these magnitudes is unknown, so I normalize the calculation to $\sim 10^{8}$ source stars. Observations of the LMC can in principle be carried out all year, but during the southern winter it is observable only at the ends of the night making simultaneous follow-up by two widely separated observatories difficult or impossible. Therefore, I assume a 
180 day summer observing season. I assume that the optical depth is $\tau \sim 2 \times 10^{-7}$ and the mean event time is $\sim 37$ days (Alcock et al. 1997b). Combining these assumptions and scaling from the previous results, I estimate there is $\sim 0.3$ EME toward the LMC per year. Moreover, in contrast to the bulge EMEs, there is little chance to measure proper motions for LMC EMEs because the sources are roughly 6 times farther away and therefore 6 times smaller. It there- fore appears that an EME search toward the LMC would not yield significant returns.

I would like to thank B. Gaudi and G. Newsom for making several valuable suggestions. Special thanks are due to Associate Editor Edward Wright who acted as referee in order to expedite the process. This work was supported in part by grant AST 94-20746 from the NSF.

\section{APPENDIX}

\section{EFFECTS OF CORRELATED NOISE}

To estimate the precision of parallax determinations in $\S 2.1$, I assumed that individual photometric measurements made once per minute had errors of $\sigma \sim 1 \%$ and that these errors were uncorrelated. In fact, a wide variety of physical processes can induce correlated errors. While several diverse observational programs are developing techniques to remove such correlated errors, this problem is substantially more difficult for extreme microlensing than for other programs and therefore warrants a separate discussion.

The quantity that one is trying to measure is the flux from the lensed star relative to an arbitrary but fixed standard. In practice, this is achieved by measuring the ratio of the flux received from the target star (TS) to the average flux from an ensemble of reference stars regarded as fixed. Thus, in principle, errors can arise either from errors in determining the flux from the TS or from errors in measuring the reference stars or from real variations in the reference stars. In fact, it is straightforward to determine if any of the reference stars are variable by comparing them with each other and (as I will discuss below) it is much easier to remove correlated errors from the reference stars than from the TS. Moreover, the total number of photons from the reference stars is much larger than the number from the TS, so they do not dominate photon statistics. For purposes of this discussion, I will therefore idealize the reference stars as forming a perfect reference standard (PRS). Many effects, such as changes in atmospheric extinction or exposure time, affect the TS and PRS in exactly the same way and therefore do not give rise to errors.

However, seeing does change the flux from the TS and PRS differently. For example, PLANET finds that for their crowded field photometry, measurements of both program and reference stars can vary by $1 \%$ or more in response to seeing changes (P. Sackett 1996, private communication). These changes can be either correlated or anticorrelated with the seeing, presumably because of faint stars moving in or out of the point spread function (PSF) as the seeing changes. For most monitoring programs, such changes pose essentially no problem, since the correlation can simply be measured and removed. Gilliland \& Brown (1992) have applied this technique to astroseismology observations of uncrowded stars and produced a photometry sequence with uncorrelated noise less than 0.1\%, i.e., much smaller than is required for EMEs. Melchior (1995) applied such corrections to pixel fluxes in crowded LMC fields and AGAPE applied them to fields of unresolved stars in M31 (J. Kaplan 1996, private communication). These latter experiences are more directly relevant to the problem at hand and I discuss them further below.

Several other physical processes can generate systematic photometry errors on various timescales, including differential refraction (diurnal), moonlight (monthly), and temperature (annual and diurnal). These effects are mediated both by the instrumentation and the data reduction process and can be quite complicated. As with problems generated by seeing variation, one can for many applications take the purely empirical approach of measuring the correlation of the photometric error with the observables and more or less blindly removing it. Unfortunately, these blind techniques cannot be applied to the analysis of EMEs.

Systematic photometry errors with power on timescales $\sim t_{\text {eff }}$ can interfere with the measurement of $\Delta t$, while those with power on scales $\gtrsim t_{\text {eff }}$ or $\gg t_{\text {eff }}$ interfere with the measurement of $\Delta \beta$. It may seem surprising that variations on timescales much longer than the observations can have any effect at all, but these long-period effects are very much at the heart of the matter. Suppose that during the Chilean winter, the TS appears systematically brighter than it would be on average during the year, while during the Australian winter it appears systematically fainter. Then the measured $\Delta \beta=\beta_{\text {Aus }}-\beta_{\text {Chi }}$ would be higher than the true value. One would like to compensate for this effect by measuring it, say by making observations of the TS during all seasons over several years. However, after the event is over, the TS is essentially gone so it is impossible to determine the correlation empirically. Effects which vary on timescales of $\sim t_{\text {eff }}$ and so affect the measurement of $\Delta t$ also cannot be calibrated empirically. Thus, one is led to ask whether these effects fundamentally compromise the proposed observations.

The answer to this question is not known. However, it is straightforward to determine unambiguously whether any given set of observations are being compromised by correlated noise. Moreover, there are indications from several types of observation that correlated noise can be reduced to a level that is close to what is required.

To determine the level of correlated noise of various types, one must monitor stable stars in the same way that one monitors microlensing events. (One must do this in any case in order to have reference stars.) If there are differences in the flux of the star (relative to the PRS) as seen from two different continents, this would reflect noise on long timescales that would compromise measurement of $\Delta \beta$. If there is time-dependent drift in the flux difference on shorter timescales, this would reflect variation on these scales. If such variations are found to lie below a given threshold for an ensemble of stable stars, then they can be assumed to lie below that threshold for the target star as well. 
Pixel lensing observations by AGAPE (Ansari et al. 1996) and Tomaney \& Crotts (1996), give a preliminary indication that correlated noise can be controlled to, or at least close to, the required level. Both groups attempt to detect microlensing events in M31, but use different techniques to reduce the data. AGAPE monitors the flux in individual pixels (or rather $7 \times 7 \sim 2^{\prime \prime} \times 2^{\prime \prime}$ super pixels). They correct for seeing variations after comparing pixel counts using a correlation technique of the type described above. Therefore, as discussed above, one cannot use these observations to test for sensitivity to seeing variation. However, one can use them to test for variations on long timescales. When they eliminate the bad-seeing data (and do not correct for seeing variation) Ansari et al. (1996) find that the superpixels with the highest photon statistics show scatter of less than $0.1 \%$ over observing campaigns lasting 5 months, a level that is comparable to what is required for monitoring EMEs. Tomaney \& Crotts (1996) convolve different images to the same seeing and then subtract one from the other, a method which could be used for EMEs (since the PSF is known from other neighboring stars). Thus, their experience is directly relevant to controlling variations in seeing. In one set of observations, they measured the flux of an apparent nova to an accuracy corresponding to $10 \%$ of the brightness of a surface brightness fluctuation and only $0.2 \%$ of the flux in a seeing disk. I find that the flux of six successive observations over $5 \mathrm{hr}$ can be fitted to a straight line with a $\chi^{2} / \mathrm{dof}=1.3$, indicating that the quoted errors are real and that the seeing-induced fluctuations are no bigger than the quoted errors. Thus, the limited experience to date indicates that the proposed observations may be feasible.

Alard, C. 1996, in IAU Symp. 173, Astrophysical Applications of Gravitational Lensing, ed. C. S. Kochanek \& J. N. Hewitt (Dordrecht: Kluwer), 215

Albrow, M., et al. 1996, in IAU Symp. 173, ed. C. S. Kochanek \& J. N. Hewitt (Dordrecht: Kluwer), 227

Alcock, C., et al. 1995a, ApJ, 445, 133

. $1995 \mathrm{~b}, \mathrm{ApJ}, 454, \mathrm{~L} 125$ .1997a, ApJ, 479, 119 1997b, ApJ, in press

Ansari, R., et al. 1996, A\&A, 314, 94

Aubourg, E., et al. 1995, A\&A, 301, 1

Baillon, P., Bouquet, A., Giraud-Héraud, Y., \& Kaplan, J. 1993, A\&A, 277, 1

Crotts, A. P. S. 1992, ApJ, 399, L43

Gaudi, B. S., \& Gould, A. 1997, ApJ, 477, 152

Gilliland, R. L., \& Brown, T. M. 1992, PASP, 104, 582

Gould, A. 1992, ApJ, 392, 442 1994a, ApJ, 421, L71 1994b, ApJ, 421, L75 1995a, ApJ, 440, 510 1995b, ApJ, 441, L21 1995 c, ApJ, 447, 491 1996a, ApJ, 470, 201 1996b, PASP, 108, 465

Gould, A., Bahcall, J. N., \& Flynn, C. 1996, ApJ, 465, 759
Gould, A., \& Loeb, A. 1992, ApJ, 396, 104

Gould, A., \& Welch, R. L. 1996, ApJ, 464, 212

Griest, K., \& Hu, W. 1992, ApJ, 397, 362

Griest, K., et al. 1991, ApJ, 372, L79

Han, C., \& Gould, A. 1995, ApJ, 447, 53 . 1996, ApJ, 467, 540

- 1997, ApJ, 480, 196

Holz, D. E., \& Wald, R. M. 1996, ApJ, 471, 64

Kiraga, M., \& Paczyński, B. 1994, ApJ, 430, 101

Light, R. M., Baum, W. A., \& Holtzman, J. A. 1996, in preparation

Mao, S., \& Paczyński, B. 1991, ApJ, 388, L45

Melchior, A.-L. 1995, Ph.D. thesis, Collège de France, Paris

Nemiroff, R. J., \& Wickramasinghe, W. A. D. T. 1994, ApJ, 424, L21

Paczyński, B. 1986, ApJ, 304, 1991, ApJ, 371, L63

Pratt, M., et al. 1996, in IAU Symp. 173, Astrophysical Applications of Gravitational Lensing, ed. C. S. Kochanek \& J. N. Hewitt (Dordrecht: Kluwer), 221

Refsdal, S. 1966, MNRAS, 134, 315

Tomaney, A., \& Crotts, A. P. S. 1996, AJ, 112, 2872

Udalski, A., et al. 1994, Acta Astron., 44, 165

Witt, H. 1995, ApJ, 449, 42

Witt, H., \& Mao, S. 1994, ApJ, 430, 505

Zhao, H. S., Spergel, D. N., \& Rich, R. M. 1995, ApJ, 440, L13 